\title{
A Recombinant Rice 16.9-kDa Heat Shock Protein Can Provide Thermoprotection in Vitro
}

\author{
Ching-Hui Yeh, Kai-Wun Yeh, Shu-Hsing Wu, Pi-Fang Linda Chang, \\ Yih-Ming Chen and Chu-Yung Lin ${ }^{1}$
}

Department of Botany, National Taiwan University, Taipei, Taiwan, R.O.C.

\begin{abstract}
It is difficult to obtain large amounts of purified low-molecular-mass heat shock proteins (LMM HSPs), which are unique to plants, for biochemical and physiological studies. Therefore, an attempt was made to produce such a HSP by applying recombinant DNA technology. We fused the cDNA for a rice class I 16.9-kDa HSP, pTSI, to the gene for glutathione S-transferase (GST) of Schistosoma japonicum and we obtained large amounts of the fusion protein from transformed Escherichia coli cells. In addition, we found that the 16.9-kDa HSP obtained by cleavage of the recombinant protein could also form a protein complex of $\sim 310 \mathrm{kDa}$ under nondenaturing conditions as can the small, native, class I HSPs from heat-shocked rice seedlings. An assay in vitro to examine the thermoprotection of rice soluble proteins from heat denaturation revealed the strong stabilizing effect of the recombinant HSP.
\end{abstract}

Key words: Expression in E. coli - Low-molecular-mass heat shock proteins - Rice Thermoprotection.

Low-molecular-mass (LMM) heat shock proteins (HSPs) are ubiquitous in plants. When exposed to a high temperature, plants produce a large and complex group of LMM HSPs that range from in molecular mass $16 \mathrm{kDa}$ to $30 \mathrm{kDa}$, in addition to high-molecular-mass (HMM) HSPs (Lindquist 1986, Vierling 1991, Yeh et al. 1994). In animals and yeasts, by contrast, the major HSPs have molecular masses of 60,70 , and $110 \mathrm{kDa}$. The induction of HSPs is assumed to provide protection from heat injury, although details of the mechanisms of action of HSPs are still poorly understood. The extent of accumulation of LMM HSPs has been found to be proportional to the severity of the heat stress. Cytological studies have shown that many small particles, known as heat shock granules (HSGs), are found as prominent new cytoplasmic structures in plants under heat stress (Nover et al. 1989). In response to heat

Abbreviations: GST, glutathion S-transferase; LMM, lowmolecular-mass; HSP(s), heat shock protein(s); HSGs, heat shock granules; IPTG, isopropylthio- $\beta$-D-galactoside; PAGE, polyacrylamidegel electrophoresis; 2-D, two-dimensional.

Supported by grants (NSC81-0211-B002-13 and NSC82-0211B002-241) to C.Y. Lin from the National Science Council of Taiwan.

1 To whom correspondence should be addressed. shock, granules of various sizes aggregate with a large amount of cytoplasmic mRNA. These observations lead to the hypothesis that the HSPs might play a role in protecting mRNA during the recovery from heat shock (HS; Nover et al. 1991). In addition, distinct HSPs were found to be localized in specific organelles, such as nuclei, mitochondria and ribosomes, and the HSPs associated with organelles shift to a soluble form under recovery conditions (Lin et al. 1984). These observations support the hypothesis that the accumulation and selective localization of LMM HSPs might provide thermoprotection to plant cells at non-permissive temperatures. A positive correlation between acquired thermotolerance in plants and the synthesis of HSPs has also been clearly demonstrated (Lin et al. 1984, Chou et al. 1989, Hsieh et al. 1992).

Genes encoding LMM HSPs have been cloned from several plant species and sequenced (Lindquist and Craig 1988, Vierling 1991). Analysis of plant genes for LMM HSP showed that they can be classified into four multigene families (I, II, III and IV), based on the localization of protein products at elevated temperatures. All known LMM HSPs are encoded by nuclear DNA and are translated by cytosolic ribosomes, and then the mature proteins are transported to the cytoplasm (class I, II), chloroplasts (class III), or endomembranes of suborganelles (class IV). The 
amino acid sequences of members of the same family of LMM HSPs include regions of high homology, designated consensus sequences I and II (Vierling 1991). This evolutionary conservation of nucleotide and amino acid sequences implies that these regions have important and fundamental physiological functions (Vierling et al. 1989).

Little is known about the biochemical and physiological characteristics of class I LMM HSPs, or about their physiological functions and mechanisms of action during thermoprotection. Immunological techniques are powerful biochemical tools with which to address these issues. However, difficulties encountered in the purification of LMM HSPs have hindered the production of significant amounts of antibody. In order to obtain sufficient LMM HSPs for immunization of rabbits, we fused the gene for glutathione S-transferase from Schistosoma japonicum (Smith et al. 1986) with the gene for a class I LMM HSP of $16.9 \mathrm{kDa}$ (Tseng et al. 1992). By inducing the tac promoter on the resultant expression plasmid, $p G T H-1$, in transformed Escherichia coli cells by treatment with isopropylthio- $\beta$ D-galactoside (IPTG), we produced large amounts of the GST-pTS1 fusion protein and the recombinant pTS1 protein was used to immunize rabbits. The preparation of antiserum recognized only class I LMM HSPs from heatshocked monocots and dicots (Jinn et al. 1993), and the antiserum also recognized the complex of native class I LMM HSPs from heat-shocked plants of several species (Jinn et al. 1995). Thermoprotection was examined in vitro as proposed by Minton et al. (1982) and we demonstrated that a single class I LMM HSP can provide thermoprotection to other soluble proteins. The nature of the pTS1 protein was also studied.

\section{Materials and Methods}

Materials-The vector $p G E X-2 T$ was purchased from Pharmacia Co. (Uppsala, Sweden). The full length cDNA clone, pTSI $(0.86 \mathrm{~kb})$, for the LMM HSP, isolated from a rice cDNA library constructed from poly (A) ${ }^{+}$mRNA of heat-stressed $41^{\circ} \mathrm{C}$ seedlings, was described previously (Tseng et al. 1992). For heat stress, three-day-old rice seedlings (Oryza sativa L. cv. Tainong 67) were incubated at $41^{\circ} \mathrm{C}$ for $2 \mathrm{~h}$.

Construction of the recombinant expression vector and induction of the LMM HSP-The expression vector used for construction of the fused gene was the Glutathione S-Transferase Gene Fusion System (GST Gene Fusion System; Pharmacia). The strategy for construction of the gene fusion is depicted in Figure 1. First, after digestion with nuclease Bal 31 (Boehringer Mannheim, Mannheim, Germany), the in-frame translatable cDNA clone, pTSI, for production of the fusion protein was isolated. The isolated cDNA fragment was modified to yield blunt ends and ligated with the $p G E X-2 T$ plasmid at a SmaI site. E. coli
XL1-blue cells were transformed with the recombinant plasmid DNA by the standard protocol (Maniatis et al. 1982). More than ten transformed clones were picked and cultured in LB broth for mini-scale preparation of DNA. DNA was sequenced to verify that it contained six nucleotides before the ATG initiation codon in a clone that was translatable in-frame.

Induction and purification of the fusion protein-Culture of cells and conditions for induction were those described previously by Smith and Johnson (1988). Cells were harvested and cell pellets were obtained by centrifugation $\left(3,000 \times \mathrm{g}, 10 \mathrm{~min}\right.$ at $\left.4^{\circ} \mathrm{C}\right)$. Pellets were resuspended in PBS buffer $\left(150 \mathrm{mM} \mathrm{NaCl}, 16 \mathrm{mM} \mathrm{Na}_{2} \mathrm{HPO}_{4}\right.$, and $4 \mathrm{mM}$ $\left.\mathrm{NaH}_{2} \mathrm{PO}_{4} \cdot 2 \mathrm{H}_{2} \mathrm{O}, \mathrm{pH} 7.8\right)$ and cells were sonicated for 15 min on ice. After centrifugation $(18,000 \times g, 15 \mathrm{~min}$ at $4^{\circ} \mathrm{C}$ ), the supernatant containing the fusion protein was collected for further purification. The fusion protein was purified in one step by passage of the supernatant through a GST-affinity gel filtration column (Pharmacia).

Characterization of the recombinant $p T S I$ proteinThe purified pTS1 fusion protein was isolated and cleaved by overnight incubation with thrombin (24 units (mg fusion protein $)^{-1}$ ) and then fragments were separated by SDS-PAGE. The recombinant pTS1 protein was eluted from the gel. The SDS associated with the eluted protein was removed by ion-pair extraction (Henderson et al. 1979). After dialysis against PBS at $4^{\circ} \mathrm{C}$ for $48 \mathrm{~h}$, the recombinant pTS1 protein was examined on a non-denaturing gradient gel ( $5 \%$ to $20 \%$ acrylamide gel).

Identification of the recombinant heat shock protein and immunization of rabbits - The recombinant pTS1 protein, prepared as described above, was sequenced on an automatic amino acid sequencer (model 477A; Applied Biosystems Inc., San Jose, CA, U.S.A.). The sequence data revealed that there are seven extra amino acid residues (derived from the thrombin-recognition sequence and from the 5 -untranslated region of $p$ TSI cDNA) before the initiation codon (ATG). The pTS1 recombinant protein $(100 \mu \mathrm{g})$ recovered from SDS-polyacrylamide gels was emulsified with Freund's complete adjuvant and injected into New Zealand rabbits to raise antiserum. The antiserum was prepared as described by Hsieh et al. (1992).

Assay for thermoprotection of soluble proteins from rice and soybean seedlings-The GST-pTS1 fusion protein or the recombinant pTS1 protein after thrombin digestion $(100 \mu \mathrm{g})$ was added to ${ }^{3} \mathrm{H}$-labeled soluble proteins $(250 \mu \mathrm{g})$ prepared from the post-ribosomal supernatant (PRS; Jinn et al. 1989) of non-heat-shocked rice and soybean seedlings. The mixture was heated at $55^{\circ} \mathrm{C}$ for $30 \mathrm{~min}$ with shaking and the denatured proteins were analyzed after pelleting by centrifugation (Jinn et al. 1989).

Electrophoresis and immunoblotting-The protein samples used in this experiment, which included fractionated LMM HSPs (precipitated between $70 \%$ and $100 \%$ 
saturation with ammonium sulfate) and the PRS, were prepared from rice seedlings by the method of Jinn et al. (1989). The protein samples were analyzed on non-denaturing gradient gels ( $5 \%$ to $20 \%$ acrylamide), by SDS-PAGE (13.75\% acrylamide) or on two-dimensional (2-D) gels by the standard protocol (O'Farrel 1975). For immunoblotting, proteins were transferred from acrylamide gels to immobilon PVDF membranes (Millipore, Bedford, CA, U.S.A.) as described by Towbin et al. (1979). The antiserum described above was used as first antibody and antibodies raised in goat against-rabbit IgG, conjugated with peroxidase (Bio-Rad, Hercules, CA, U.S.A.), were used as second antibody. The conditions of immuno-reactions were those described by Bio-Rad.

\section{Results}

Overexpression of the inducible GST-pTSI fusion protein-A cDNA for a 16.9-kDa LMM HSP of rice, $p T S 1$, was inserted into the $p G E X-2 T$ vector to yield $p G T H-I$ (Fig. 1), for production of the GST-pTS1 fusion protein. Theoretically, the fusion protein consisted of the glutathione S-transferase protein, with six amino acid residues specific for digestion by thrombin (L-V-P-R-G-S), and the pTS1 protein which contained seven extra amino acids (GS-P-I-P-A-T) before the first methionine residue. E. coli cells containing the $p G T H-1$ plasmid were grown overnight in LB/Ampicillin broth, and induced to produce the fusion protein by addition of IPTG (final concentration, $1 \mathrm{mM}$ ). Cells were harvested after two hours of incubation with IP. TG and lysates were analyzed by SDS-PAGE. E. coli cells that had been transformed with the $p G T H-1$ plasmid produced high levels of the fusion protein. The maximum yield was $10 \mathrm{mg}$ per liter. The fusion protein was further purified by affinity chromatography on a column of Sepharose CL4B-GST. The recombinant pTS1 protein was generated by incubation with thrombin, purified by SDS-PAGE and eluted from gels (Fig. 2). A significant amount of an almost homogeneous preparation of a 17.6-kDa LMM HSP was obtained. The amount of GST protein (indicated by an open triangle in Fig. 2) was much higher than that of recombinant pTS1 protein (indicated by a filled triangle), as deduced from the intensity of protein bands on a Coomassie blue-stained gel (Fig. 2, lane 4). Furthermore, some other bands of protein were detected by the SDSPAGE after purification on the affinity column (Fig. 2, lanes 3 and 4). To establish the purity of the recombinant pTS1 protein, the peptide sequences of eluted samples were analyzed. As shown in Table 1, the peptide sequence of recombinant pTSl was the same as that deduced from pTS1 cDNA, except for the seven extra residues. Moreover, the observed $\mathrm{pI}$ of the recombinant pTS1 protein was similar to the value for $\mathrm{pTS} 1$ protein deduced from $p T S 1$ cDNA.
Specificity of antiserum against pTS1 HSP and characterization of the recombinant pTSI HSP-In previous study, the specificity of the antiserum was examined by Western blotting, and we found that it recognized only class I LMM HSPs from heat-shocked plants (Jinn et al. 1993). When we used the antiserum to examined the recombinant pTS1 protein, as shown in Figure 2B, a 17.6$k D a$ protein and several other proteins were recognized but the GST protein was not. For identification of the recombinant 16.9-kDa HSP from rice seedlings, the HSP-enriched fraction from heat-shocked rice seedlings was fractionated with ammonium sulfate (70\% to $100 \%$ saturation) and subjected to SDS-PAGE (as shown by Hsieh 1990 and Jinn et al. 1993) or subjected to electrophoresis on a non-denaturing gradient gel ( $5 \%$ to $20 \%$ acrylamide; Jinn et al. 1995)

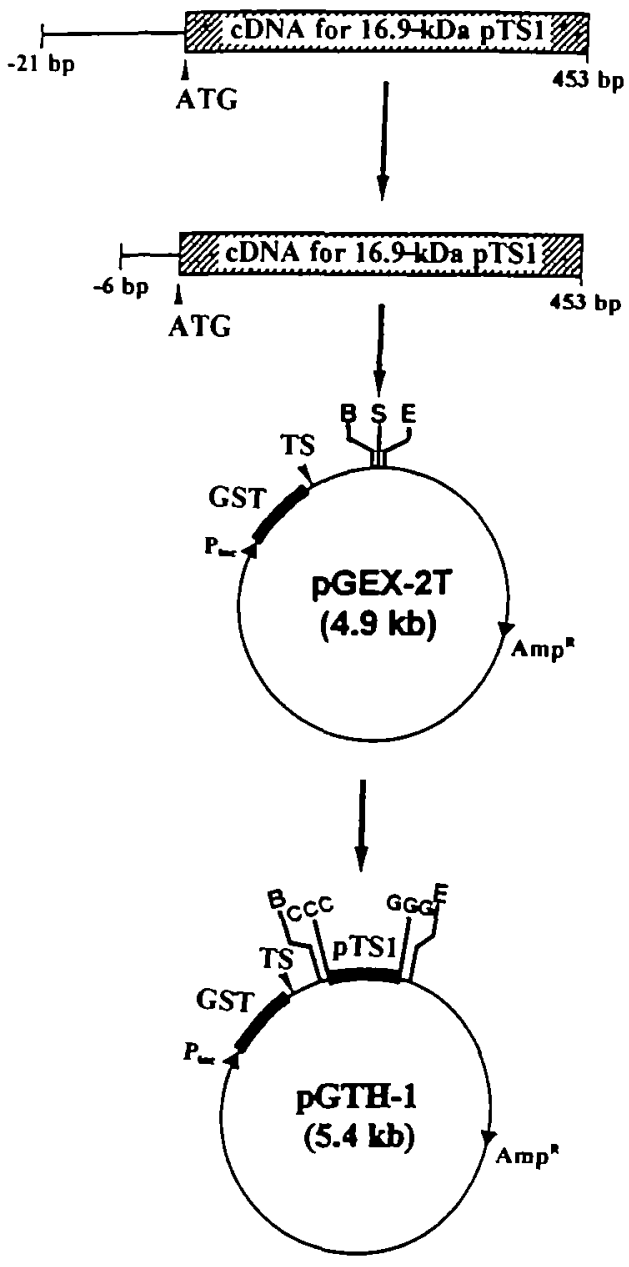

Fig. 1 Construction of the GST-pTSI fusion gene. A 474 bp blunt-ended cDNA fragment encoding the 16.9-kDa LMM HSP of rice was inserted into the $p G E X-2 T$ expression vector at the SmaI site to yield $p G T H-1$. Amp ${ }^{\mathrm{R}}$, Ampicillin-resistance gene; GST, gene for glutathione S-transferase (Smith et al. 1986); B, $B a m H I, ~ S, S m a I, E, E c o R I ; T S$, thrombin-specific cleavage site. 


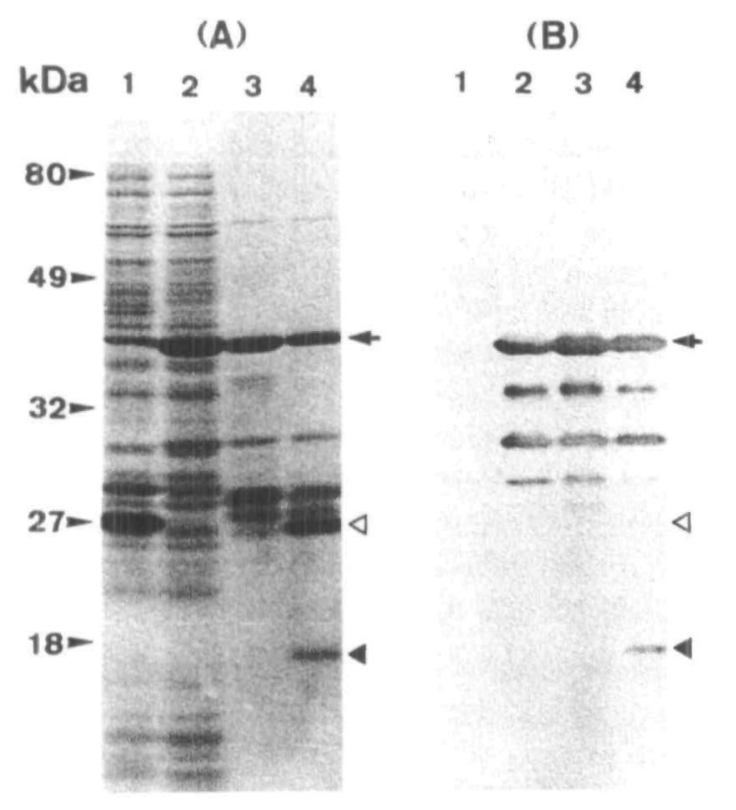

Fg. 2 Expression and purification of the rice pTS1 fusion protein produced by $E$. coli cells and immunological reactivity of the recombinant pTS1 protein with antiserum raised against recombinant pTS1. E. coli XL1-blue cells were transformed with $p G T H-l$ and the fusion protein was obtained after induction by IPTG for $2 \mathrm{~h}$. Protein samples (10 $\mu \mathrm{g}$ each) were subjected to SDS-PAGE (13.75\% acrylamide). (A) Coomassie blue-stained gel after SDS-PAGE. (B) Western blot with pTS1-specific antiserum. Lane 1 , control sample, namely, total proteins from $E$. coli cells transformed with the $p G E X-2 T$ plasmid (no insert); lane 2, total proteins from $E$. coli cells that produced the fusion protein GSTpTS1; lane 3, fusion protein purified by affinity chromatography on a column of glutathione Sepharose $4 B$; lane 4 , purified fusion protein after cleavage with thrombin. An arrow indicates the GST-pTSI fusion protein $(45.1 \mathrm{kDa})$, an open triangle indicates the GST protein $(27.5 \mathrm{kDa})$, and a filled triangle indicates the recombinant pTS1 protein $(17.6 \mathrm{kDa})$.

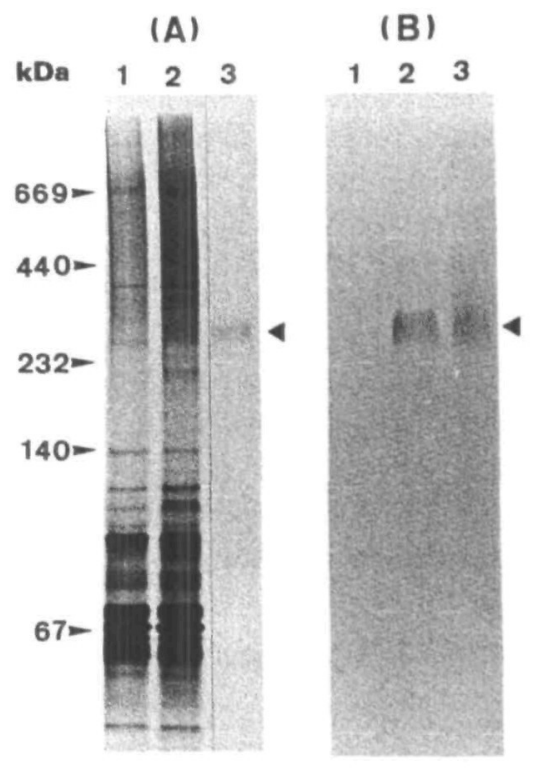

Fl. 3 Identification and immunological reactivity of the HSP complex with pTS1-specific antiserum. Proteins were extracted from normally grown $\left(28^{\circ} \mathrm{C}\right)$ and heat-shocked $\left(41^{\circ} \mathrm{C}, 2 \mathrm{~h}\right)$ rice seedlings and fractionated with ammonium sulfate (70\% to $100 \%$ saturation). (A) Silver-stained non-denaturing gradient gel after PAGE; (B) Western blot with pTS1-specific antiserum. A filled triangle indicates the $\sim 310 \mathrm{kDa}$ complex (lanes 2 and 3). Lane 1 , proteins from normally grown seedlings; lane 2 , proteins from heat-shocked seedlings; lane 3, gel-purified recombinant pTS1 protein after removal of SDS and dialysis against PBS (as described in Materials and Methods) to yield the native form.

for Western blotting. A protein complex of $\sim 310 \mathrm{kDa}$ was evident on the non-denaturing gradient gel (Figs. 3A, B, lane 2). This complex was recovered from the gel and fur-

Table 1 Characteristics of the recombinant pTS1 protein obtained from the thrombin-treated GST-pTS1 fusion protein that was expressed from $E$. coli, in a comparison with those of the protein deduced from the nucleotide sequence of $p$ TSI cDNA

\begin{tabular}{|c|c|c|}
\hline Characteristic & Recombinant pTSI ${ }^{a}$ & Deduced pTS1 ${ }^{b}$ \\
\hline N-terminal sequence & $\begin{array}{l}\text { G-S-P-I-P-A-T-M*-S-L-V-R-R-S-N-V-F-D-P-F } \\
\text { (peptide determination) }\end{array}$ & $\begin{array}{l}\text { M*-S-L-V-R-R-S-N-V-F-D-P-F } \\
\text { (cDNA prediction) }\end{array}$ \\
\hline Molecular mass & $17.6 \mathrm{kDa}$ & $16.9 \mathrm{kDa}$ \\
\hline Amino acid residues & 157 & 150 \\
\hline $\mathrm{pI}$ & 6.4 & 6.4 \\
\hline \multicolumn{3}{|c|}{$\begin{array}{l}\text { The data were obtained from the peptide sequence, determined with an automated sequencer, and the molecular mass and pl } \\
\text { deduced from mobilities on gels. }\end{array}$} \\
\hline
\end{tabular}


ther analyzed on 2-D gels for identification of the $16.9-\mathrm{kDa}$ LMM HSP in this complex (Fig. 4). The recombinant pTS1 protein recovered from SDS-containing gels after dialysis in PBS for $48 \mathrm{~h}$ formed a large native complex on nondenaturing gradient gels. Immunostaining demonstrated that the protein sample formed a complex of $\sim 310 \mathrm{kDa}$, namely, of the same size as the complex of LMM HSPs in vivo (Figs. 3A, B, lane 3). The recombinant pTS1 expressed in prokaryotic $E$. coli cells was, thus, also able to form multimers, which might be important for physiological function. When the recombinant pTS1 protein after thrombin digestion was mixed with rice LMM HSPs or the native complex and then subjected to 2-D PAGE, it migrated with one of the members of the HSP family (Fig. 5). As we predicted previously, pTS1 belongs to the class of the most abundantly expressed genes in the group of rice class I LMM HSPs since the spot $(17.5 \mathrm{kDa}, \mathrm{pI} 6.4)$ that corresponded to pTS1 (16.9 kDa, pI 6.4) was more prominent than others.

Thermoprotection of soluble proteins against heat

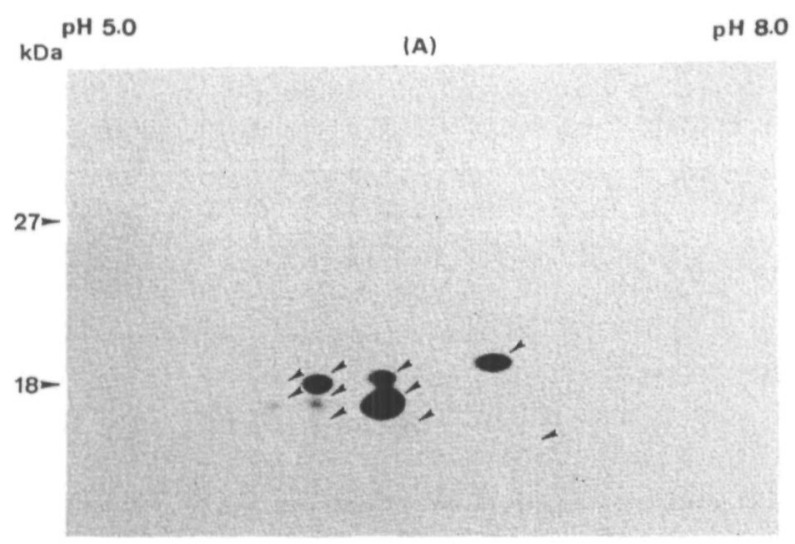

(B)

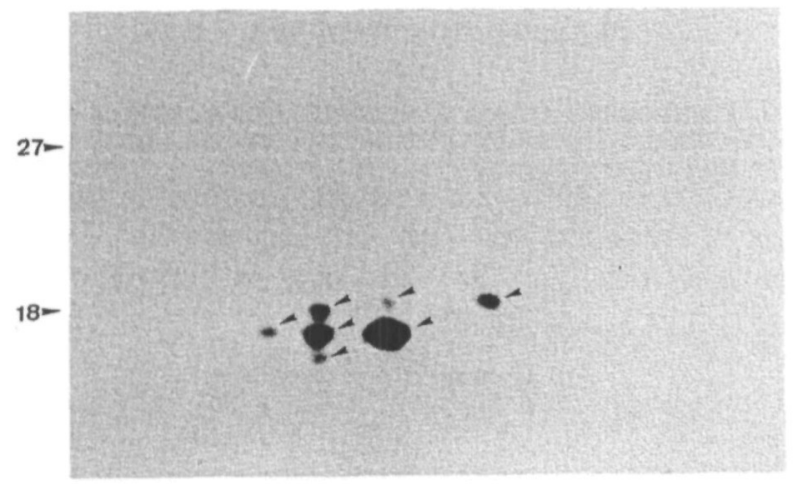

Fig. 4 Immunological identification of rice LMM HSPs with pTS1-specific antiserum. The protein complex of $\sim 310 \mathrm{kDa}$ (as shown in Fig. 3A, lane 2) was eluted from the gel and subjected to 2-D PAGE. (A) Silver-stained gel. (B) A subsequent Western blot with pTS1-specific antiserum. denaturation by the recombinant pTS1 protein-Previous studies showed that HSP-enriched fractions (obtained between $70 \%$ and $100 \%$ saturation with ammonium sulfate), prepared from heat-shocked seedlings of rice and three dicots (soybean, mung bean and pea), could thermostabilize the homologous soluble proteins (Jinn et al. 1989). Up to $50 \%$ of the soluble proteins that were normally denatured by heating at $55^{\circ} \mathrm{C}$ for $30 \mathrm{~min}$ were protected when a HSP-enriched fraction was added to the soluble proteins from any of the four plant species tested. These observations strongly suggest that the thermostabilizing functions of HSPs toward cellular soluble proteins are effective across a variety of plant species. Moreover, they highlight the importance of the conserved structures of LMM HSPs, even in highly divergent plant species. The pTS1 fusion protein and the recombinant pTS1 protein were used in assays of thermostabilization in vitro. Such assays were perform-

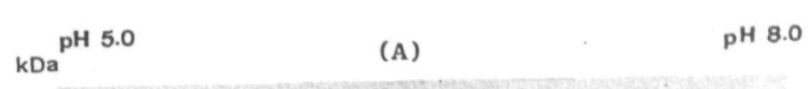

(B)

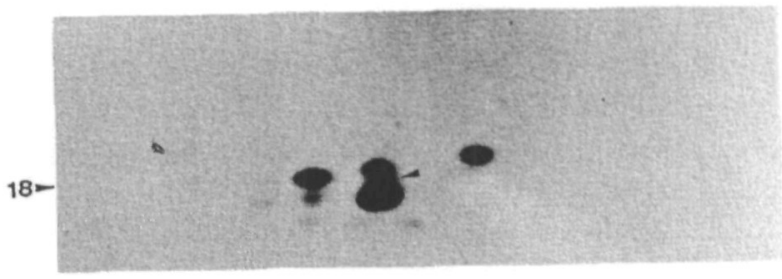

(c)

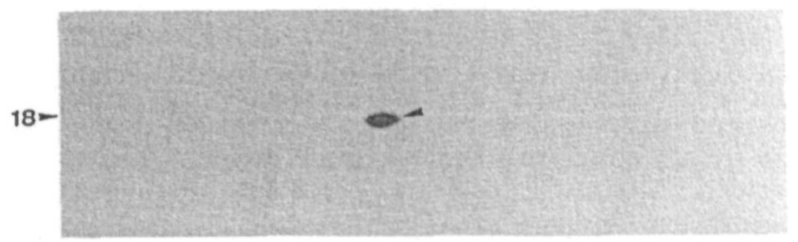

Fig. 5 Identification of pTS1 as a member of the rice LMM HSP gene family. Recombinant pTSI protein was mixed with the LMM HSP complex, which had been recovered as the protein complex of $\sim 310 \mathrm{kDa}$ from a non-denaturing gradient gel (as shown in Fig. 3A, lane 2), and the mixture was separated by 2-D PAGE to determine the location of the recombinant PTS1 protein by silver staining. (A) The $\sim 310-\mathrm{kDa}$ protein complex alone, (B) the $\sim 310-k D a$ protein complex plus gel-purified recombinant pTS1 protein, and $(C)$ the gel-purified recombinant pTSI protein alone. The position of recombinant pTSI is indicated by an arrowhead. 
Table 2 Thermostabilization of ${ }^{3} \mathrm{H}$-labeled soluble proteins prepared from postribosomal supernatant (PRS) of rice and soybean seedlings by the GST-pTS1 fusion protein and the recombinant pTS1 protein obtained after digestion with thrombin

\begin{tabular}{|c|c|c|}
\hline \multirow{2}{*}{ Addition } & \multicolumn{2}{|c|}{ Denatured soluble proteins (cpm) } \\
\hline & Rice & Soybean \\
\hline Control (70\%-100\% $\left(\mathrm{NH}_{4}\right)_{2} \mathrm{SO}_{4}$ saturation) & $21,000(100 \%)$ & $73,000(100 \%)$ \\
\hline HSP-enriched fraction $\left(70 \%-100 \%\left(\mathrm{NH}_{4}\right)_{2} \mathrm{SO}_{4}\right.$ saturation) & $15,000 \quad(70 \%)$ & $51,000 \quad(72 \%)$ \\
\hline GST-pTS1 fusion protein & $16,000 \quad(75 \%)$ & $59,000(80 \%)$ \\
\hline Recombinant pTS1 protein (thrombin-digested) & $12,000 \quad(58 \%)$ & $44,000 \quad(60 \%)$ \\
\hline
\end{tabular}

To $250 \mu \mathrm{g}$ of ${ }^{3} \mathrm{H}$-labeled proteins in the postribosomal supernatant $\left(5.5 \times 10^{4} \mathrm{cpm}\right)$, we added $100 \mu \mathrm{g}$ of the $70 \%-100 \%$ ammonium sulfate fraction from seedlings of rice or soybean, the GST-pTS1 fusion protein, or the recombinant pTS1 protein after thrombin digestion. Each mixture was incubated at $55^{\circ} \mathrm{C}$ for $30 \mathrm{~min}$. After heat treatment, each mixture was centrifuged at $16,000 \times g$ for 15 min. The pellets were suspended in $250 \mu \mathrm{l}$ of Laemmli's sample buffer and assayed for radioactivity. Control: the reaction mixture was supplemented with the HSP-enriched fraction (70\%-100\% ( $\left(\mathrm{NH}_{4}\right)_{2} \mathrm{SO}_{4}$ saturation), prepared from 4-day-old seedlings grown at $28^{\circ} \mathrm{C}$. HSP-enriched fraction: the protein sample was prepared as in the control, except the seedlings were incubated at $41^{\circ} \mathrm{C}$ for $2 \mathrm{~h}$ (rice) or $40^{\circ} \mathrm{C}$ for $2 \mathrm{~h}$ (soybean) before extraction of protein. The radioactivity of tritiated protein that was pelleted after heating is the mean value from triplicate samples from at least two separate experiments (S.D. was less than 5\%).

ed previously with the HSP-enriched protein fractions from seedlings that had been heat-shocked at $41^{\circ} \mathrm{C}$ (Jinn et al. 1989). The pTS1 fusion protein and the recombinant pTS1 protein obtained from the fusion protein by treatment with thrombin were added to ${ }^{3} \mathrm{H}$-labeled PRS fractions. These mixtures were heated at $55^{\circ} \mathrm{C}$ for $30 \mathrm{~min}$ and the radioactivity in denatured proteins was then determined. As shown in Table 2, the recombinant pTS1 protein provided effective thermoprotection to soluble proteins, as did the pTS1 fusion protein and the HSP-enriched fraction from heat-shocked seedlings. pTS1 also protected the proteins in the samples from soybean (Wu 1992).

\section{Discussion}

E. coli cells that had been transformed with the $p G T H-1$ plasmid produced high levels of GST-pTS 1 fusion protein. After purification on a GST-affinity column, more than one protein was present in the purified sample of GST-pTS1 protein (Fig. 2A, lane 3). When this protein sample was treated with thrombin, some proteins with molecular masses different from those of GST-pTS1, GST, and pTS1 proteins were detected after SDS-PAGE (Fig. 2A, lane 4). Furthermore, the molar ratio of GST to pTS1 should have been 1:1 after thrombin digestion but, as shown in Fig. 2A (lane 4), the amount of pTS1 was less than that of GST. However, the protein bands appeared in Figure 2A (lanes 3 and 4) were also recognized by the pTS1specific antiserum (Fig. 2B, lanes 3 and 4). We suggest that fusion proteins of various sizes were probably the result of incomplete translation of the pTS1 protein by $E$. coli for some reason, so that various proteins with molecular masses larger than $27.5 \mathrm{kDa}$ (the molecular mass of GST) all cross-reacted with the pTS1-specific antiserum. Moreover, $20 \%$ to $30 \%$ of the $E$. coli cells died upon treatment with IPTG in our experiments (data not shown) which might explain the production of fusion proteins of different lengths. Thus, after digestion of fusion proteins with thrombin, the ratio of levels of pTS1 $(17.6 \mathrm{kDa})$ to GST $(27.5 \mathrm{kDa}$ ) seen in Figure 2A (lane 4) was not $1: 1$ because small, incompletely translated fragments of pTS1 might have run off the gel. The mono-specific polyclonal antisera raised against the soybean class I LMM HSPs (Hsieh et al. 1992) and against recombinant pTSI protein only recognized the class I LMM HSPs of rice and the other plant species examined (Jinn et al. 1993). No cross reaction was observed with proteins in a lysate of $E$. coli cells (Fig. 2B). Some proteins of more than $27.5 \mathrm{kDa}$ that were recognized by the antiserum might have been in some conformation that prevented thrombin from cleaving the peptides completely.

The antiserum raised against the recombinant pTS1 protein identified multimeric native protein complex in an extract (70\% to $100 \%$ saturation with ammonium sulfate) of heat-shocked rice seedlings. The complex had an apparent molecular mass of $\sim 310 \mathrm{kDa}$, as determined on non-denaturing gradient gels (Fig.3). A similar protein complex was also found in other plant species, such as, soybean, mung bean, and pea (Jinn et al. 1995). All of these complexes were strongly cross-reactive with the antiserum raised against rice pTS1 (data not shown).

According to Jinn et al. (1995), the LMM HSPs (as multimeric native protein complexes of 250 to $310 \mathrm{kDa}$ in extracts of rice and other dicot plants) play an important role in protecting soluble proteins from heat denaturation. It will be of interest to determine whether the formation of 
multimeric complexes by these LMM proteins is necessary for thermoprotection. The rice protein complex of $\sim 310$ $\mathrm{kDa}$ was composed mainly of $16-$ to $18-\mathrm{kDa}$ proteins, according to the pattern after SDS-PAGE (Fig. 4A), in agreement with the composition of the in vitro hybrid-selected products of translation of pTS1 poly(A) ${ }^{+}$RNA from heatshocked rice seedlings (Tseng et al. 1993). In order to identify pTS1 in the protein complex, the recombinant pTS1 protein (which has the same $\mathrm{pl}$ and a similar molecular mass to those predicted from the deduced amino acid sequence of pTSI cDNA shown in Table 1), was mixed with the protein complex that had been recovered from the gel after 2-D gel analysis. The spot of pTS1 could be distinguished from those of other LMM HSPs; it is indicated by an arrowhead in Figure 5B and was identified from the position of the recombinant pTS1 protein shown in Figure 5C. Although the protein complex of $\sim 310 \mathrm{kDa}$ was composed of LMM HSPs of 16-18 kDa (Figs. 4A, 5A) which included pTS1, the recombinant pTS1 protein alone, after recoverey from gels after SDS-PAGE, retained the ability to form a protein complex of $\sim 310 \mathrm{kDa}$ (Figs. 3A, B, lane 3) after removal of SDS. We estimate that the $\sim 310-\mathrm{kDa}$ protein complex of rice shown in Figure 3A (lane 2) consisted of about 15-17 subunits of 16.9-kDa LMM HSPs.

The recombinant pTS1 protein protected soluble proteins from heat denaturation $\left(55^{\circ} \mathrm{C}\right)$ in vitro as did the native class I LMM HSP complex (Jinn et al. 1995). Furthermore, the recombinant pTS1 protein not only protected rice soluble proteins from heat denaturation but it also protected soybean soluble proteins (Table 2). The thermoprotective function of recombinant LMM HSPs is, thus, exchangeable among different plant species, as we showed previously with an HSP-enriched fraction (Jinn et al. 1993). The thermoprotection in vitro by recombinant pTS1 clearly supports the hypothesis that the LMM HSPs have an important physiological function. We are now testing the recombinant pTS1 protein expressed in E. coli for its thermoprotective effects.

\section{References}

Chou, M., Chen, Y.M. and Lin, C.Y. (1989) Thermotolerance of isolated mitochondria associated with heat shock proteins. Plant Physiol. 98: 617-621.

Henderson, L.E., Oroszlan, S. and Konigsberg, W. (1979) A micro method for complete removal of dodecyl sulfate from proteins by ion-pair extraction. Anal. Biochem. 93: 153-157.

Hsieh, M.H. (1990) Preparation and characterization of soybean LMM HSP-specific antibody. Master thesis. Dept. of Botany, National Taiwan University, Taipei, Taiwan, Republic of China.

Hsieh, M.H., Chen, J.T., Jinn, T.L., Chen, Y.M. and Lin, C.Y. (1992) A class of soybean low-molecular-mass heat shock pro- teins. Plant Physiol. 99: 1279-1284.

Jinn, T.L., Chen, Y.M. and Lin, C.Y. (1995) Characterization and physiological function of class I low-molecular-mass heat shock protein complex in soybean. Plant Physiol. 108: 693-701.

Jinn, T.L., Wu, S.H., Yeh, C.H., Hsieh, M.H., Yeh, Y.C., Chen, Y.M. and Lin, C.Y. (1993) Immunological kinship of class I low-molecular-mass heat shock proteins and thermostabilization of soluble proteins in vitro among plants. Plant Cell Physiol. 34: 1055-1062.

Jinn, T.L., Yeh, Y.C., Chen, Y.M. and Lin, C.Y. (1989) Stabilization of soluble proteins in vitro by heat shock proteins-enriched ammonium sulfate fraction from soybean seedlings. Plant Cell Physiol. 30: 463-469.

Lin, C.Y., Roberts, J.K. and Key, J.L. (1984) Acquisition of thermotolerance in soybean seedlings. Plant Physiol. 74: 152160.

Lindquist, S. (1986) The heat-shock response. Annu. Rev. Biochem. 45: 39-72.

Lindquist, S. and Craig, E.A. (1988) The heat shock proteins. Annu. Rev. Genet. 22: 631-677.

Maniatis, T., Fristch, E.F. and Sambrook, L. (1982) Molecular Cloning: a Laboratory Manual. Cold Spring Harbor, New York.

Minton, K.W., Karmin, P., Hahn, G.H. and Minton, A.P. (1982) Nonspecific stabilization of stress-resistant proteins: a model for the biological roles of heat shock proteins. Proc. Natl. Acad. Sci. USA 79: 7107-7111.

Nagao, R.T., Czarnecka, E., Gurley, W.B. and Key, J.L. (1985) Genes for low-molecular-mass heat shock proteins of soybeans: sequence analysis of a multigene family. Mol. Cell Biol. 5: 3417-3428.

Nover, L., Neumann, D. and Scharf, K.D. (1991) Intracellular localization and related functions of heat shock proteins. In Heat Shock Response. Edited by Nover, L. pp. 373-408. CRC Press, Ann Arbor, Michigan.

Nover, L., Scharf, K.D. and Neumann, D. (1989) Cytoplasmic heat shock granules are formed from precursor particles and are associated with a specific set of mRNAs. Mol. Cell. Biol. 9: 1298-1308.

O'Farrel, P.H. (1975) High resolution two-dimension electrophoresis of proteins. J. Biol. Chem. 10: 4007-4021.

Smith, D.B., Davern, K.M., Board, P.G., Tiu, W.U., Garcia, E.C. and Mitchell, G.F. (1986) Mr 26,000 antigen of Schistosoma japonicum recognized by resistant WEHI $129 / \mathrm{J}$ mice is a parasite glutathione S-transferase. Proc. Natl. Acad. Sci. USA 83: 8703-8707.

Smith, D.B. and Johnson, K.S. (1988) Single-step purification of polypeptides expressed in Escherichia coli as fusions with glutathione S-transferase. Gene 67: 31-40.

Towbin, T.S., Staehelin, T. and Gordon, J. (1979) Electrophoretic transfer of protein from polyacrylamide gels to nitrocellulose sheet: procedure and some application. Proc. Natl. Acad. Sci. USA 76: 4350-4354.

Tseng, T.S., Tzeng, S.S., Yeh, K.W., Yeh, C.H., Chang, F.C., Chen, Y.M. and Lin, C.Y. (1993) The heat shock response in 
rice seedlings: isolation and expression of cDNAs that encode class I low-molecular-weight heat-shock proteins. Plant Cell Physiol. 34: 165-168.

Tseng, T.S., Yeh, K.W., Yeh, C.H., Chang, F.C., Chen, Y.M. and Lin, C.Y. (1992) Two rice (Oryza sativa) full-length cDNA clones encoding low-molecular-weight heat shock proteins. Plant Mol. Biol. 18: 963-965.

Vierling, E. (1991) The role of heat shock proteins in plants. Annu. Rev. Plant Physiol. Plant Mol. Biol. 42: 579-620.

Vierling, E., Harris, L.M. and Chen, Q. (1989) The major lowmolecular-weight heat shock protein in chloroplasts shows an- tigenic conservation among diverse plant species. Mol. Cell. Biol. 9: 461-468.

Wu, S.H. (1992) The biological function of low-molecular-mass heat shock proteins in plants. Master thesis. Dept. of Botany, National Taiwan University, Taipei, Taiwan, Republic of China.

Yeh, K.W., Jinn, T.L., Yeh, C.H., Chen, Y.M. and Lin, C.Y. (1994) Plant low-molecular-mass heat-shock proteins: their relationship to the acquisition of thermotolerance in plants. Biotechnol. Appl. Biochem. 19: 41-49.

(Received March 13, 1995; Accepted August 17, 1995) 